\title{
DRUG-RELATED PNEUMONIA DUE TO CLOZAPINE TREATMENT
}

\section{Serhat TUNC ${ }^{1}$, Hamit Serdar BASBUG ${ }^{2}$}

\section{${ }^{1}$ Department of Psychiatry, Kafkas University, Kars, TURKEY \\ YI E PA ${ }^{2}$ Department of Cardiovascular Surgery, Kafkas University, Kars, TURKEY}

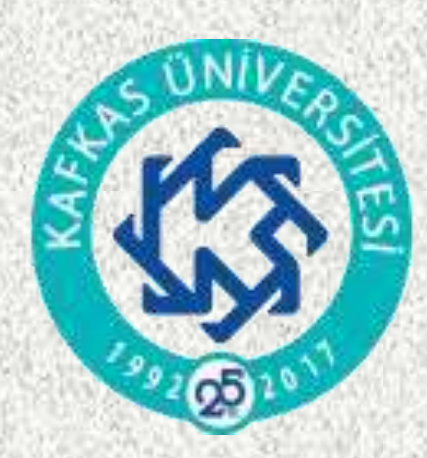

Drug-related pneumonia is common mainly due to acid reducing agents, antipsychotic drugs, glucocorticoids, and sedatives. Clozapine is an extremely efficient antipsychotic and the single antipsychotic with an indication for suicidal behavior and treatment-resistant schizophrenia. However several side effects have been related to clozapine use. An increased risk of lower respiratory infections, including pneumonia was reported in individuals using atypical antipsychotics. Among the atypical antipsychotics, clozapine is related to the highest risk. We state that clozapine may cause aspiration pneumonia in schizophrenia patient.

A 28-year-old female admitted with a purulent cough, fever, shortness of breath, and fatigue for the last eight days. In her drug history clozapine, $200 \mathrm{mg} /$ day usage was detected for the last two years with a diagnosis of schizophrenia. She had never experienced a pulmonary complication before. Her cough and fatigue were getting worse for the last three days. The biochemical test revealed elevated white blood cells, erythrocyte sedimentation rate, and C-reactive protein. Chest computed tomography (CT) demonstrated multifocal infiltrates revealing for an infectious etiology. The sputum culture revealed a gram-positive bacteria.

She was hospitalized and given intravenous medication with the diagnosis of pneumonia. The clozapine dose was reduced to $100 \mathrm{mg}$ /day from 200 $\mathrm{mg} /$ day. She recovered in two days. The general condition of the patient was improved.

Clinicians should keep in mind that both the infection and the drug treatment of the infection by drug-drug interactions at CYP1A2 can cause elevated and toxic levels of serum clozapine that may lead to more troubles.

Keywords: clozapine, adverse drug event, pneumonia 